\title{
DISTAL URETERAL STUMP: CASE REPORT
}

\section{Bulotta AL, Ferrara F, Sica M, Di Maggio G, Messina M}

\section{Division of Pediatric Surgery, Department of Pediatrics, Obstetrics and Reproductive Medicine University Of Siena}

Introdution. Distal Ureteral Stump is a residual ureter after total or partial nephrectomy. It is a rare complication and it also appears many years after surgery. Majority of patients are asymptomatic but Literature reports patients with recurrent bacteriuria or haematuria, empyema, stones and tumors (transitional-cell carcinoma or renal-cell carcinoma). We present one case of diseased ureteral stump and surgical strategy.

Case Report. We report a case of a patient subjected to retroperitoneoscopic total nephrectomy when he was seven months old for sympthomatic right vesico-ureteral reflux of IV grade and associated renal hypoplasia. These patient presented recurrent urinary infections after 3 years from the surgery and for this reason we performed voiding micturating cystourethrography who revealed the presence of urinary reflux in the DUS. Because of this surgical removal of stump was necessary and during 2 months follow-up was normal and there weren't surgical complications or UTI.

Discussion. Distal Ureteral Stump is a rare complication but possible after nephrectomy and it is due to partial excision of ureter in the distal portion. Recurrent urinary infections are a usefull signal to subspect the presence of DUS and they are due to persistent reflux of urine and dysfunctional voiding (reservoir) resulting in stasis and infections.

Therefore in all patients subjected to total or partial nephrectomy with recurrent urinary infections also after years, the presence of DUS should always be suspected and radiological investigation must be performed for accurate management. Keywords: nephroureterectomy, distal ureteral stump, vesico-ureteral reflux, stumpectomy.

\section{INTRODUCTION}

Classically the management of vesico-ureteral reflux associated to poorly functioning kidney is nephrectomy and total removal of ureter. However total removal of ureter is not always possible, especially if it is ectopic. For this reason a distal ureteral stump (DUS) may remain. Literature reports minimal late complications in DUS but when pathologic findings occur it is necessary a patient's surveillance and appropriate therapeutic strategy.

Generally the diagnosis of DUS is clinical for presence of infections, stones, tumors and obstruction with or without concomitant infections. In others patients it is described the "Stump Ureteral Syndrome" characterized by recurrent urinary infection, abdominal pain and haematuria. Radiologic support is necessary to confirm the diagnosis and a simple mintional retrograde cystouretrography can see the stump and its morphology if refluxing.

We present an assessment of patient with DUS post total nephrectomy and our diagnostic and surgiacal approach.

\section{CASE REPORT}

M.S, a male born at term by natural childbirth after a normal pregnancy.

Fetal ultrasound didn't show abdominal malformations. After the birth the baby presented many episodes characterized by fever, abdominal pain and urine culture showed the presence of many colonies of Escherichia Coli. Also the patient showed important growth retardation. For this reason when he was three months of live abdominal ultrasound was performed and it revealed a right renal hypoplasia (diameter 2,5 $\mathrm{cm}$ ) and reduction of renal medulla while the left kidney (diameter $6,5 \mathrm{~cm}$ ) was normal. Scintigraphy with DMSA confirmed the right renal hypoplasia and we performed a voiding micturating cystourethrography that showed a right grade 4 vesico-ureteral reflux.

When he was old 7 months of life right retroperitoneoscopic nephrectomy was performed with removal of ureter by an additional incision on the flank. The histological examination confirmed the renal hypoplasia and dysplasia with disorganization of muscle fibers of pieloureteral junction and ureter. The patient was discharged in seventh day and there weren't complications like fever, obstruction or UTI.

However the patient presented recurrent urinary infections after 4 years from the surgery and antibiotic therapy was prescribed to the baby.

We performed voiding micturating cystourethrography that it showed the presence of urinary reflux in the right distal ureteral stump also after total bladder empty (Fig.l a- b). Because the parents did not accept to continue antibiotic therapy surgical removal of stump was necessary. 
Fig.1. Voiding micturating cystourethrography a) distal ureteral stump after total bladder filling; b) DUS after voiding phase.

a

\section{SURGICAL TREATMENT}

Cystoscopy was performed With the patient in the dorsal lithotomy position. It revealed the presence of normal left ureter and right ureter with stadium forms who ended at dead end. After having positioned Fogarty's catheter (Chatelier 6) in DUS to allow a simple identification of it during the surgery, the cystoscopy was extract.

Through Pfannenstiel incision, without enter in peritoneal cavity, bladder was individualized and then DUS was identified back and isolated from all bystanders anatomical structures like vas deferens.

Identification of DUS was facilitaded by Fogarty's catheter inside. Therefore DUS was closed at the base by purse-string suture and excised before removing the catheter (Fig. 2 a-b).

After control of haemostasis, the surgical breack was sutured in layers. Foley's catheter was positioned in the bladder to avoid overfilling and it was removed after 3 days.

The patient was discharged in fifth day and he took antibiotic for one week. There weren't complications like fever, obstruction or UTI. During 2 months follow-up was normal and there weren't surgical complications or UTI.

\section{DISCUSSION}

Nephrectomy is currently the treatment of choise in patients with vesico-ureteral reflux associated with hypoplasia and reduced renal function. In fact, is often useless to try to save a kidney against endoscopic suburetereal injection or bladder' surgery. Many technique are described to perform nephrouretectomy from open approach to laparoscopic or retroperitoneoscopic surgery. Therefore one of the measures of nephroureterectomy is the complete removal of ureter at its base to reduce risk of a distal ureteral stump and urinary infections (1). Because of this Literature recommends dual approach with complete excision of the kidney and ureter (2). We performed for this reason an incision on the flank but ureter was ectopic and it is impossible a complete excision of it. Many studies about refluxing ureteral stump in a duplicated system suggest that an ectopic ureteral stump does not necessarily predispose to complications like recurrent urinary infection and then stumpectomy is not always necessary.

In fact the ureteral stump left after total or partial nephrec- tomy for primary vesicoureteral reflux presents a low rate of complications even when there is a high-grade reflux.

Cain et al. reviewed the outcome of 38 refluxing ureteric stumps after nephrectomy and hemi- nephrectomy, with a follow-up of up to 13 years, and found that only two patients $(5 \%)$ required a secondary procedure to remove the DUS because of breakthrough UTIs. These two patients were girls with additional risk factors for UTI, i.e. contralateral reflux and ureterocele (3). Androulakakis et al. showed their longterm experience with the outcome of both refluxing and nonrefluxing DUS. Only one of 89 patients re- 
quired a secondary operation to remove the DUS, because of recurrent episodes of haematuria and bacteriuria despite antibiotic treatment (5).

Pathogenicity of DUS is due to its poor peristaltic activity, which don't prevents urinary stasis. Because of this a long refluxing stump inevitably acts as a diverticulum from which urine cannot be effectively drained. Another significant observation is the role of dysfunctional voiding in causing acquired reflux into a long DUS, leading to urinary stasis and infection (3, 4). This occurred many years after partial ureterectomy contradicts the view that the lumen of ureteric stumps becomes occluded by fibrosis soon after the operation. The complications of DUS are not only recurrent urinary infections but also empyema, stones and tumors (transitional-cell carcinoma or renal-cell carcinoma) (6, $7)$. Therefore the surgical removal of the symptomatic DUS must always be perfomed in this patient because it is safe, it leads to a regression of symptoms and reduced the risk of much more serious complications. Although there have been reported cases in which the resolution of DUS was performed by endoscopic electrofulguration, occlusion of the stump with Teflon paste (8) or laparoscopic management, we preffered a little Pfannenstiel incision. In fact we have obtained a good exposition of ureteral stump without opening the abdominal cavity and with the safety to completely resect the stump. So our patient has been feed immediately and we minimized the risk of recurrence of stump. In conclusion, when of distale ureteral stump is suspected, all patients presenting recurrent urinary infection after a nephroureterectomy, must undergo a carefull radiological investigation particulary the voiding micturating cystourethrography.

\section{REFERENCES}

1. Persad R, Kamineni S, Mouriquand PD: Recurrent symptoms of tract infection in eight patients with refluxing ureteric stumps. Br J Urol 1994; 74: $720-722$

2. Krarup T, Wolf H. Refluxing ureteral stump. Scand J Urol Nephrol 1978; 12:181-183.

3. Cain MP, Pope JL, Casale AJ, Adams MC, Keating MA, Rink RC. Natural history of refluxing distal ureteral stumps after nephrectomy and partial ureterectomy for vesicoureteral reflux. J Urol 1998; 160: 1026-7. 4. Amar A. Refluxing ureteral stump: Reservoir of urinary tract infection. J Urol 1964; 1:493-495.

5. Androulakakis PA, Stephanidis A, Antoniou a, Christophoridis C. Outcome of the distal ureteric stump after (hemi)nephrectomy and subtotal ureterectomy for reflux or obstruction. BJU International 2001; 88: 586589

6. Prajsner A, Szewczyk W, Gawron R. Empyema of Retained Ureteral Stump With Gross Stone 20 Years After Nephrectomy. J Urol 2009; 73. 993-994.

7. Park JY, Park J, Ku JH, Kim HH. Transitional Cell Carcinoma in a Remnant Ureter after Retroperitoneoscopic Simple Nephrectomy for Benign Renal Disease. J Korean Med Sci 2009; 24: 992-4.

8. Bullock KN, Deane AM, Ashken MH. Endoscopic Teflon injection for a refluxing ureteric stump after simplenephrectomy. British Medical Journal 1985; 290: 1009

Fig.2. Stumpectomy after Pfannenstiel incision:

a) DUS was identified and isolated from all bystanders anatomical structure;

b) After closure of DUS by purse-string suture, it is removed at the base.
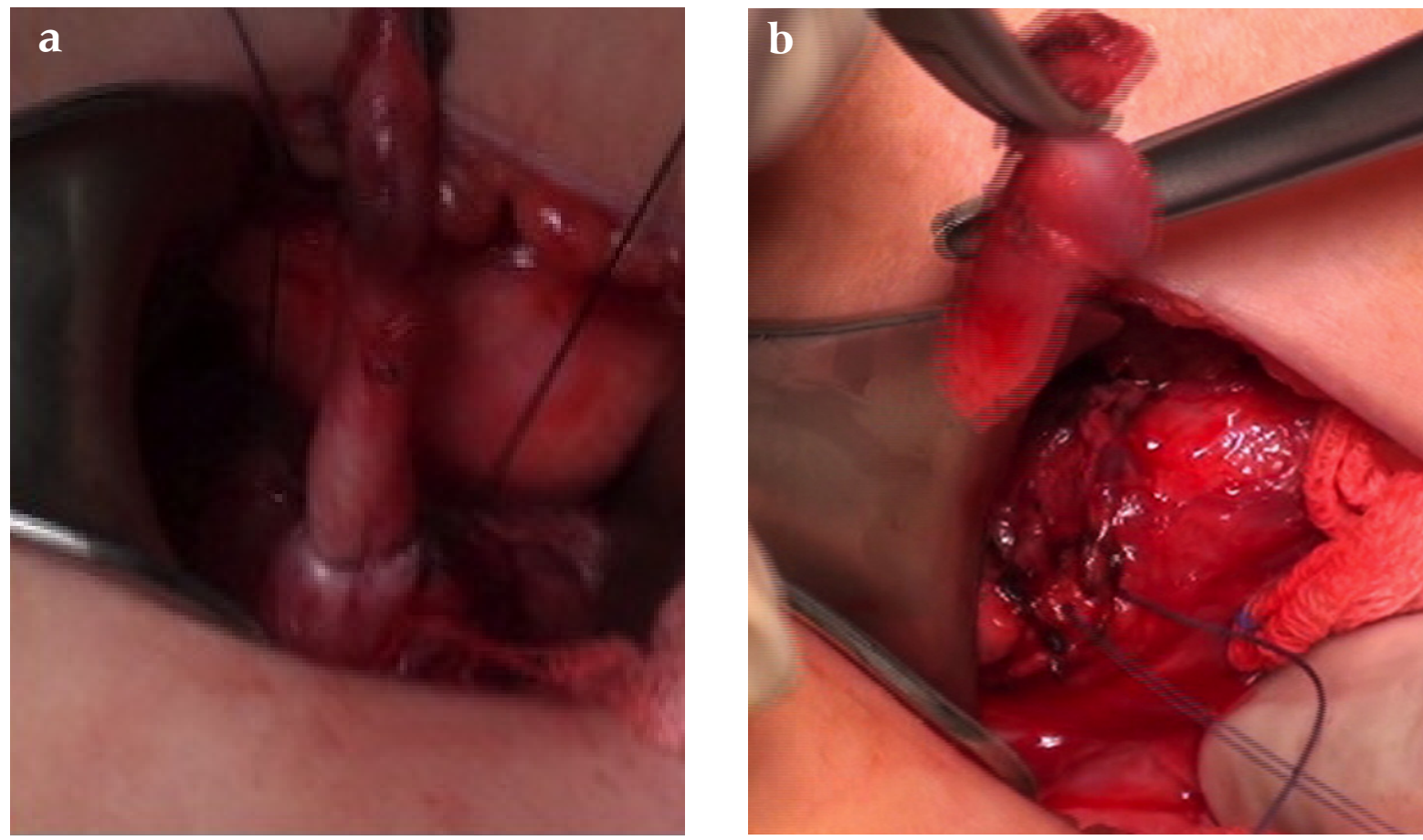